\title{
Web report European Society of Cardiology educational website
} Vinay K Sharma

\author{
Attending Physician, Pulmonary, Critical Care and Sleep Medicine Division, Graduate Hospital, Philadelphia, Pennsylvania, USA \\ Correspondence: Vinay K Sharma, vinayks@yahoo.com
}

Published online: 21 November 2003

Critical Care 2004, 8:70 (DOI 10.1186/cc2397)

This article is online at http://ccforum.com/content/8/1/70

(c) 2004 BioMed Central Ltd (Print ISSN 1364-8535; Online ISSN 1466-609X)

Website European Society of Cardiology educational website

URL www.esced.org

Cost Currently free

Keywords cardiology, critical care

ESCed is an educational website developed by the European Society of Cardiology. The site is currently free and access is obtained after registering with the website. The educational content is based on the format of case presentations.

The site presently has 18 modules grouped into seven categories: acute coronary syndromes, chronic ischaemic heart disease, heart failure, arrhythmias, hypertension, valve disease and coronary heart disease risk factors. The website states that more modules are under development.

Each module starts with a short case history followed by a series of multiple-choice questions. The questions progress in chronological order and ask the user to select investigations or interventions at critical points as the patient's clinical history unfolds. The time since symptom onset and/or the time since admission is listed on each question page. At the top of each question page are icons that display the medical history, clinical notes and test results. Images are provided for tests such as electrocardiography, echocardiography, angiography or computed tomography. Short video clips can also be viewed for echocardiography and angiography. On the answer page a bar chart displays how often each option presented in the question has been selected by previous users. Comments are made regarding each of the options and references are cited. Selecting a reference displays the abstract of that article. When reference is made to a European Society of Cardiology guideline, a link is provided to view the full text document.

Most pages have a box with further information related to the case being presented; for example, 'other causes of dyspnoea' in a case presenting with dyspnoea, 'biochemical markers of myocardial necrosis' in a patient presenting with chest pain or 'CABG versus medical therapy' in a patient with chronic stable angina. All drugs mentioned in the modules are highlighted, and selecting a drug opens a popup box with information about the drug, including indications, formulation, cautions, contraindications, side effects, dose and drug interactions. This information is taken from the
Some of the modules contain logos of pharmaceutical companies. However, the exact role of and the extent of the involvement of these companies has not been declared on the website. A few modules have received support from the European Union.

ESCed is accredited for continuing medical education by the European Board for Accreditation in Cardiology, which works in cooperation with the European Accreditation Council for Continuing Medical Education. At the end of each module the user can obtain 1 hour of continuing medical education credit.

Entering a keyword or phrase in the search engine displays the relevant case histories and the cited references. The site also has a discussion forum that currently has eight topics, including aspirin in patients with acute coronary syndrome and peptic ulcer, and haemodynamic instability in atrial fibrillation. However, very few comments have so far been posted.

Overall, I found the cases to be well presented with a wealth of related information presented in each module. I think medical students and residents will obtain great educational value from visiting the site. In addition to physicians with an interest in cardiology, the site should also be of interest to emergency room physicians, intensivists and general medicine physicians. The number of topics covered is presently limited, but the site can only get better as more modules are added.

\section{Best feature}

A wealth of information in each module.

\section{Worst feature}

A lack of links to other websites.

\section{Wish list}

Interactive clinical scenarios where the case is allowed to progress with the intervention you choose with a possible negative outcome.

\section{Competing interests}

None declared. 